\section{Diferenciais intraurbanos da sífilis congênita:
análise preditiva por bairros do Município do \\ Diferenciais intraurbanos da sífilis congênita:
análise preditiva por bairros do Município do Rio de Janeiro, Brasil}

\author{
Intraurban differentials in congenital syphilis: a \\ predictive analysis by neighborhood in the city of \\ Rio de Janeiro, Brazil
}

\section{Diferenciales intraurbanos de sífilis congénita: análisis predictivo por barrios del municipio de Río de Janeiro, Brasil}

Gilson Jácome dos Reis 1

Christovam Barcellos 2

Marcel de Moraes Pedroso 2

Diego Ricardo Xavier 2 doi: 10.1590/0102-311X00105517

\section{Resumo}

O objetivo deste estudo foi caracterizar os casos notificados de sifilis congênita no período de 2011 a 2014, no Município do Rio de Janeiro, e analisar possíveis associações entre a morbidade por sífilis congênita e as condições de vida das populações residentes nos bairros da cidade. Os casos de sífilis congênita foram caracterizados de acordo com variáveis biológicas, socioeconômicas e de utilização de serviços de saúde. No nível agregado, utilizou-se a árvore de regressão como técnica de análise de dados, tendo a taxa de incidência média (2011-2014) de sífilis congênita como variável dependente, e indicadores relativos à qualidade habitacional, educação, renda, gravidez na adolescência, densidade de pobres, acesso à assistência pré-natal e cor da pele como variáveis independentes. Houve mapeamento da variável dependente para a identificação de padrões espaciais. Utilizaram-se dados do SINAN, SINASC e IBGE. Foram notificados 6.274 casos de sífilis congênita, o que representa uma taxa de incidência de 17,3 casos/mil nascidos vivos. Os casos se distribuem preponderantemente na zona central, norte suburbana e oeste da cidade, com elevada proporção de casos com baixa escolaridade e de cor da pele negra. Observou-se alta proporção de gestantes que tiveram diagnóstico tardio de sifilis e tratamento inadequado. No nível agregado, a variável mais relevante para a explicação dos problemas foi a baixa proporção de gestantes que frequentaram, no minimo, sete consultas de assistência pré-natal. A análise permitiu a identificação de segmentos de populações marginalizadas, podendo direcionar de maneira efetiva a distribuição de recursos de saúde pública.

\author{
Correspondência \\ G. J. Reis \\ Escola Nacional de Saúde Pública Sergio Arouca, Fundação \\ Oswaldo Cruz. \\ Rua Leopoldo Bulhões 1480, Rio de Janeiro, RJ 21041-210, \\ Brasil. \\ gilson_jacome@yahoo.com.br \\ 1 Escola Nacional de Saúde Pública Sergio Arouca, Fundação \\ Oswaldo Cruz, Rio de Janeiro, Brasil. \\ 2 Instituto de Comunicação e Informação Científica e \\ Tecnológica em Saúde, Fundação Oswaldo Cruz, Rio de Janeiro, \\ Brasil.
}




\section{Introdução}

A sífilis é uma doença infectocontagiosa sistêmica, de evolução crônica, causada pela bactéria Treponema pallidum. Sua transmissão pode ocorrer por via sexual, vertical e sanguínea. A sífilis congênita é resultado da disseminação hematogênica do agente etiológico da gestante infectada não tratada ou inadequadamente tratada para o seu concepto 1 .

As medidas de controle da sífilis congênita - diagnóstico e tratamento oportuno da sífilis em gestante e no(s) parceiro(s) sexual(is) - são efetivas para evitá-la. A despeito disso, a sífilis apresenta as maiores taxas de transmissão vertical dentre as diversas doenças que podem ser transmitidas durante o ciclo grávido-puerperal 2. Estima-se que em termos globais, em 2008, houve 576.784 casos de sífilis congênita 3. Em 2012, a incidência reduziu de maneira expressiva, sendo estimados 350.915 casos, o que representa uma redução de 39\%. A África e o Sudeste Asiático foram as regiões com as incidências mais elevadas, sendo que no Sudeste Asiático foi onde se observou a redução mais expressiva no referido período, sobretudo na Índia. Na região das Américas também houve redução do número de casos, com exceção do Brasil.

Apesar da redução global da incidência da sífilis congênita e do fato de o Sistema Único de Saúde (SUS) disponibilizar testes diagnósticos e o tratamento para as gestantes, no contexto da assistência pré-natal - considerada a medida de Saúde Pública mais efetiva para o controle da sífilis congênita sua incidência permanece com valores bastante elevados no Brasil. Segundo dados do Ministério da Saúde 4, a taxa de incidência da sífilis congênita, em 2015, foi de 6,5 casos a cada mil nascidos vivos, o que representa, em termos absolutos, 19.235 novos casos de sífilis congênitas no país. No Estado do Rio de Janeiro a situação é ainda mais grave. A taxa de incidência foi a mais elevada dentre todas as Unidades da Federação em 2015, atingindo 12,4 casos/mil nascidos vivos 4. No Município do Rio de Janeiro, a magnitude da doença foi ainda maior, atingindo o valor de 17,4 casos/mil nascidos vivos em 2015.

Algumas abordagens do problema da sífilis congênita vêm sugerindo que devido à sua magnitude ela seja utilizada como um dos indicadores para o monitoramento da qualidade da atenção materno-infantil. Saraceni et al. 5 constataram que a despeito da redução geral da mortalidade perinatal no Município do Rio de Janeiro, entre 1999 e 2002, o indicador de mortalidade perinatal específica por sífilis congênita não apresentou redução, e desta maneira sugere-se que estes casos sejam utilizados como eventos sentinela para avaliação da qualidade da atenção à mulher e à criança ${ }^{5}$. Em um estudo sobre a prevalência da sífilis congênita em âmbito hospitalar, De Lorenzi \& Madi ${ }^{6}$ constataram problemas no diagnóstico e tratamento da sífilis em gestantes, ressaltando a importância da incidência de sífilis congênita como indicador de qualidade da assistência pré-natal ${ }^{6}$. Num estudo de avaliação da assistência pré-natal na prevenção da sífilis congênita, Domingues et al. 7 apontaram diversas questões na resposta ao problema, definindo a sífilis congênita como evento sentinela da qualidade da assistência pré-natal 7 .

Lima et al. 8, num estudo transversal de avaliação da qualidade do rastreamento do HIV/AIDS e sífilis na assistência pré-natal em Salvador, Bahia, Brasil, evidenciaram que apesar da cobertura da assistência pré-natal ter sido superior a 90\%, entre 2005 e 2006, houve desigualdade na qualidade da assistência prestada. As parcelas populacionais de renda baixa têm acesso a um serviço de pior qualidade, pois iniciam o acompanhamento pré-natal tardiamente, realizam menor número de consultas e têm menor chance de realizar o rastreamento da sífilis no primeiro trimestre da gestação 8 .

Estudos ecológicos evidenciaram que a incidência da sífilis congênita é mais elevada em estratos populacionais de menor escolaridade, em grupos raciais desfavorecidos socioeconomicamente (negros) e com piores condições de vida 9,10,11. Em abordagens cuja unidade de análise é o indivíduo, observou-se maior risco de ocorrência da sífilis congênita em negros, dentre aqueles cuja mãe é parda ou negra e que possui baixa escolaridade, e entre os de menor status econômico 8,12.

As abordagens sobre o processo saúde/doença/cuidado, em nível populacional, podem ser favorecidas pelo potencial explicativo do território, apreendido como o resultado de acumulações históricas, que proporciona condições para a ocorrência de doenças, consistindo em uma categoria de síntese e convergência de processos relacionados à saúde, condições de vida e ambiente 13 .

Este trabalho propõe-se a compreender as características da transmissão vertical da sífilis, as desigualdades espaciais de sua incidência nos bairros do Município do Rio de Janeiro, bem como as possíveis associações com as variáveis relacionadas às condições de vida e de utilização dos serviços 
de saúde, oferecendo subsídios ao planejamento das ações de resposta, com a finalidade de controlar a transmissão vertical da sífilis.

\section{Métodos}

Ainda que o estudo presuma o processo de determinação social dos fenômenos de saúde e doença, o presente trabalho está voltado para o resultado deste processo, isto é, um retrato da situação da doença e seus determinantes, expresso no período de 2011-2014. A utilização da média do quadriênio 2011-2014 para cálculo dos indicadores contribui para a estabilização das taxas das unidades de análise agregadas. A agregação desse período é uma forma de evitar que flutuações aleatórias de alguns indicadores com números absolutos muito pequenos interfiram nos resultados do estudo. Os dados utilizados são oriundos do Sistema de Informação de Agravos de Notificação (SINAN), do Sistema de Informações sobre Nascidos Vivos (SINASC) (Secretaria Municipal de Saúde do Rio de Janeiro, TABNET municipal. http://tabnet.rio.rj.gov.br) e do Censo Demográfico de 2010 do Instituto Brasileiro de Geografia e Estatística (IBGE. Censo Demográfico 2010. https://www.ibge.gov.br/estatisticasnovoportal/sociais/saude/9662-censo-demografico-2010.html?=\&t=o-que-e).

Os casos notificados de sífilis congênita foram caracterizados de acordo com variáveis relativas ao local de residência, condição socioeconômica e de utilização dos serviços de saúde por parte da mãe.

A definição dos bairros da cidade do Rio de Janeiro como unidades espaciais de análise ancorou-se em alguns critérios 14, quais sejam: disponibilidade de informações sobre a localização dos indivíduos doentes e expostos (SINAN e SINASC), bem como de variáveis relativas às condições de vida das populações; reconhecimento da população em relação a seus bairros de residência; e o fato de o bairro consistir em território de atuação do estado e de grupos populacionais organizados.

Buscou-se realizar a análise dos problemas de saúde/doença de maneira articulada ao território, usando-se técnicas de geoprocessamento, com o emprego do Sistema de Informação Geográfica (ArcGIS - http://www.esri.com/software/arcgis/index.html). As malhas cartográficas digitais do Município do Rio de Janeiro foram adquiridas no sítio eletrônico do IBGE, e elas dividem a cidade em 161 bairros, incluindo o bairro da Lapa, criado por meio da Lei Municipal no 5.407/2012. Como as bases de dados do SINAN e SINASC (2011-2014) não incluem a Lapa como bairro de residência, este bairro não foi objeto de análise. Os bairros de Parque Colúmbia, Vasco da Gama e Gericinó são relativamente recentes, e por isto não dispõem de reconhecimento social de seus habitantes, provocando problemas nos processos de notificação e registro e, consequentemente, na análise dos dados. Por isso, tais bairros foram agregados aos bairros da Pavuna, São Cristóvão e Bangu, respectivamente. Dessa forma, o total de unidades de análise do estudo foi de 157 bairros.

No que diz respeito aos indicadores utilizados na análise, buscou-se selecionar um que expressasse a magnitude do problema (variável-desfecho) e aqueles que poderiam explicar o problema, de acordo com a perspectiva teórica adotada. A Tabela 1 apresenta os indicadores usados, sua dimensão e fonte de dados.

A taxa de incidência de sífilis congênita foi calculada por meio da divisão do número de casos notificados, em menores de 1 ano, pelo número de nascidos vivos residentes nos bairros da cidade do Rio de Janeiro, no período de 2011-2014, e multiplicado por mil. Esse indicador estima a intensidade com que a doença acomete a população residente no período e espaço definidos 15, e foi mapeado para a identificação da distribuição espacial da ocorrência de novos casos de sífilis congênita na cidade do Rio de Janeiro.

As variáveis investigadas sobre as condições de vida das populações residentes nos bairros do Município do Rio de Janeiro foram definidas com base nas evidências existentes, e condicionadas à disponibilidade de dados. Algumas variáveis foram selecionadas por comporem o Índice de Desenvolvimento Social (IDS), inspirado no Índice de Desenvolvimento Humano (IDH). O cálculo do IDS inclui, além dos aspectos relacionados ao desenvolvimento humano, variáveis relacionadas às condições de vida em ambiente urbano 16.

O número de banheiros por moradores é uma variável que estima a qualidade de habitação e compõe o Índice de Desenvolvimento Social (IDS). É calculado por meio da divisão do número de banheiros no domicílio particular permanente pelo número total de pessoas no domicílio particular. 
Tabela 1

Indicadores utilizados na análise do problema.

\begin{tabular}{|c|c|c|}
\hline Dimensão do indicador & Indicadores & Fontes \\
\hline Magnitude do problema & Taxa de incidência média de 2011-2014 de sífilis congênita & SINAN e SINASC \\
\hline \multirow[t]{2}{*}{ Qualidade habitacional } & Número de banheiros por morador, em 2010 & IBGE \\
\hline & (BANMOR) & \\
\hline Educação & Proporção de analfabetismo na população de 10-14 anos, em 2010 (ANALF1014) & IBGE \\
\hline \multirow[t]{2}{*}{ Renda } & Rendimento médio dos responsáveis (que têm rendimento) pelos domicílios em & IBGE \\
\hline & salários mínimos, em 2010 (RENDMSALM) & \\
\hline \multirow[t]{2}{*}{ Gravidez na adolescência } & Proporção de gravidez na adolescência, média de 2011 a 2014 & SINASC \\
\hline & (GRAVIDEZADOLESC) & \\
\hline Pobreza & Densidade populacional de pobres, em 2010 (DENSPOBRE) & IBGE \\
\hline Utilização da assistência pré-natal & Proporção de nascidos vivos cuja mãe fez 7 consultas ou mais no pré-natal, & SINASC \\
\hline & média de 2011 a 2014 (PRENATAL7MAIS) & \\
\hline \multirow[t]{2}{*}{ Cor da pele } & Proporção da população cuja cor da pele é parda, 2010 (PPPAR) & IBGE \\
\hline & Proporção da população cuja cor da pele é preta, 2010 (PPPRE) & IBGE \\
\hline
\end{tabular}

IBGE: Instituto Brasileiro de Geografia e Estatística; SINAN: Sistema de Informação de Agravos de Notificação; SINASC: Sistema de Informações sobre Nascidos Vivos.

Nota: elaborado pelos autores.

A taxa de analfabetismo na população entre 10 e 14 anos foi o indicador selecionado para mensurar a educação no IDS. Diante da ausência da variável escolaridade (número de anos de estudos) no Censo de 2010, selecionou-se a variável analfabetismo. A definição do intervalo de 10-14 anos de idade ocorreu porque o fenômeno apresenta maior variabilidade neste grupo populacional. $\mathrm{O}$ rendimento médio dos responsáveis pelos domicílios em salários mínimos estima a renda e compõe o IDS de 2010. A proporção de nascidos vivos cuja mãe é adolescente é calculada por meio da divisão dos nascidos vivos cujas mães tinham entre 10 e 19 anos, sobre o total de nascidos vivos residentes nos bairros do Rio de Janeiro, no período de 2011-2014. A proporção de nascidos vivos cuja mãe fez sete consultas ou mais no pré-natal estima a cobertura de serviços de saúde voltados para a assistência pré-natal. É calculada por meio da divisão do número de nascidos vivos cuja mãe frequentou sete consultas ou mais pelo número total de nascidos vivos. A densidade populacional de pobres foi calculada usando-se a divisão do número de moradores em domicílios particulares permanentes, com rendimento nominal mensal da pessoa responsável pelo domicílio, com renda entre zero e dois salários mínimos (proveniente do Censo IBGE de 2010), pela área útil dos bairros em $\mathrm{km}^{2}$. Esse indicador estima a concentração de pessoas pobres no território. A proporção da população cuja cor da pele é parda e a proporção daqueles cuja cor da pele é preta são indicadores calculados pela divisão do número de habitantes de cor parda ou preta pelo número total de habitantes nos bairros do Rio de Janeiro, no ano de 2010. Para a verificação do grau de correlação entre as variáveis explicativas e a variável desfecho, utilizou-se o coeficiente de correlação de Spearman.

Para analisar as relações entre a variável desfecho e as variáveis explicativas adotou-se o modelo denominado árvore de decisão, técnica útil para a identificação das variáveis contínuas mais relevantes para a descrição de um problema. O método utiliza um algoritmo para análise de dados capaz de se adaptar e ser sensível a diferentes distribuições do conjunto de dados, e tem sido usado nas análises de dados em saúde 17. O algoritmo selecionado - CaRT (Classification and Regression Trees) - é um modelo de regressão que estabelece relação com as variáveis explicativas e apenas uma variável contínua de desfecho, de modo que haja divisões do conjunto de dados (nós da árvore), criando subconjuntos de dados com valores da variável desfecho homogêneos. Para a avaliação do modelo, o conjunto de dados foi subdividido em dois grupos aleatórios, um grupo de treino (com $\mathrm{x} \%$ dos bairros) e um grupo de teste (com os $\mathrm{x} \%$ restantes). Após a construção da árvore foi analisado o erro médio quadrático nas estimativas das taxas, e a comparação entre os dois conjuntos de dados possibilitou o cálculo do erro absoluto médio no modelo. A representação das árvores de decisão tem uma estrutura hierárquica, 
apresentando em sua parte superior o conjunto total de dados, que é dividido progressivamente na direção inferior, de acordo com as premissas do algoritmo, desempenhando uma função de classificação e previsão facilmente interpretável 18. Os resultados finais calculam a taxa com base nas variáveis explicativas e o intervalo de confiança para as previsões estimadas. A denominação árvore de decisão, para tal técnica de análise, é genérica; quando se usam variáveis contínuas - situação do presente estudo - a denominação específica é árvore de regressão ${ }^{19}$. Foi utilizado o software R, incluindo o pacote RPART (The R Foundation for Statistical Computing, Viena, Áustria; http://www.r-project. org), para a operacionalização da análise.

\section{Resultados}

De 2011-2014 foram notificados 6.274 casos de sífilis congênita, em menores de 1 ano, considerandose os residentes na cidade do Rio de Janeiro. Em relação às variáveis socioeconômicas e de utilização dos serviços de saúde, a Tabela 2 apresenta a distribuição proporcional dos casos notificados de sífilis congênita no Município do Rio de Janeiro.

\section{Tabela 2}

Distribuição proporcional dos casos notificados de sífilis congênita no Município do Rio de Janeiro, Brasil, cujo diagnóstico ocorreu no período de 2011-2014.

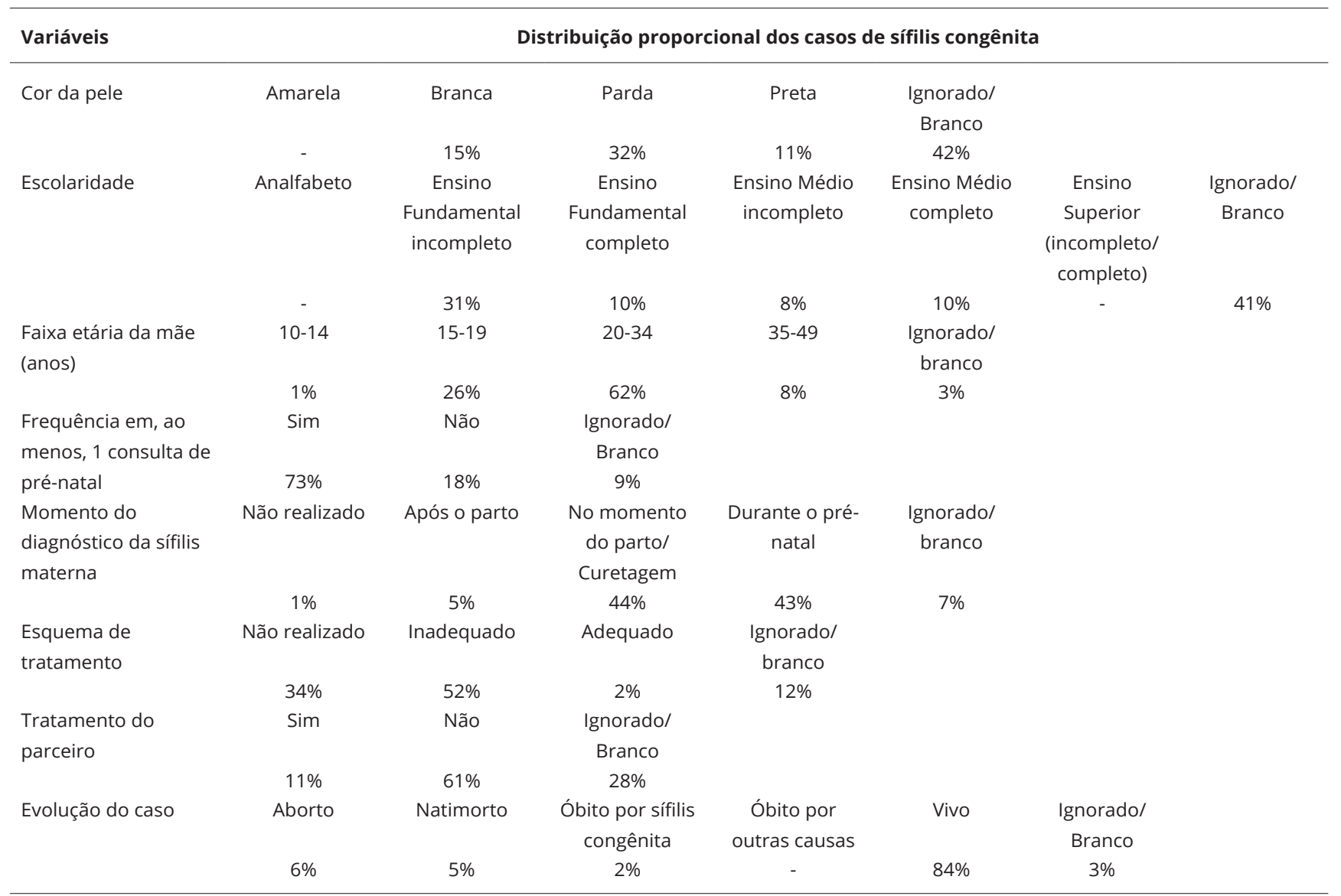

Fonte: Sistema de Informação de Agravos de Notificação, Secretaria Municipal de Saúde do Rio de Janeiro. Elaboração própria. 


\section{Figure 1}

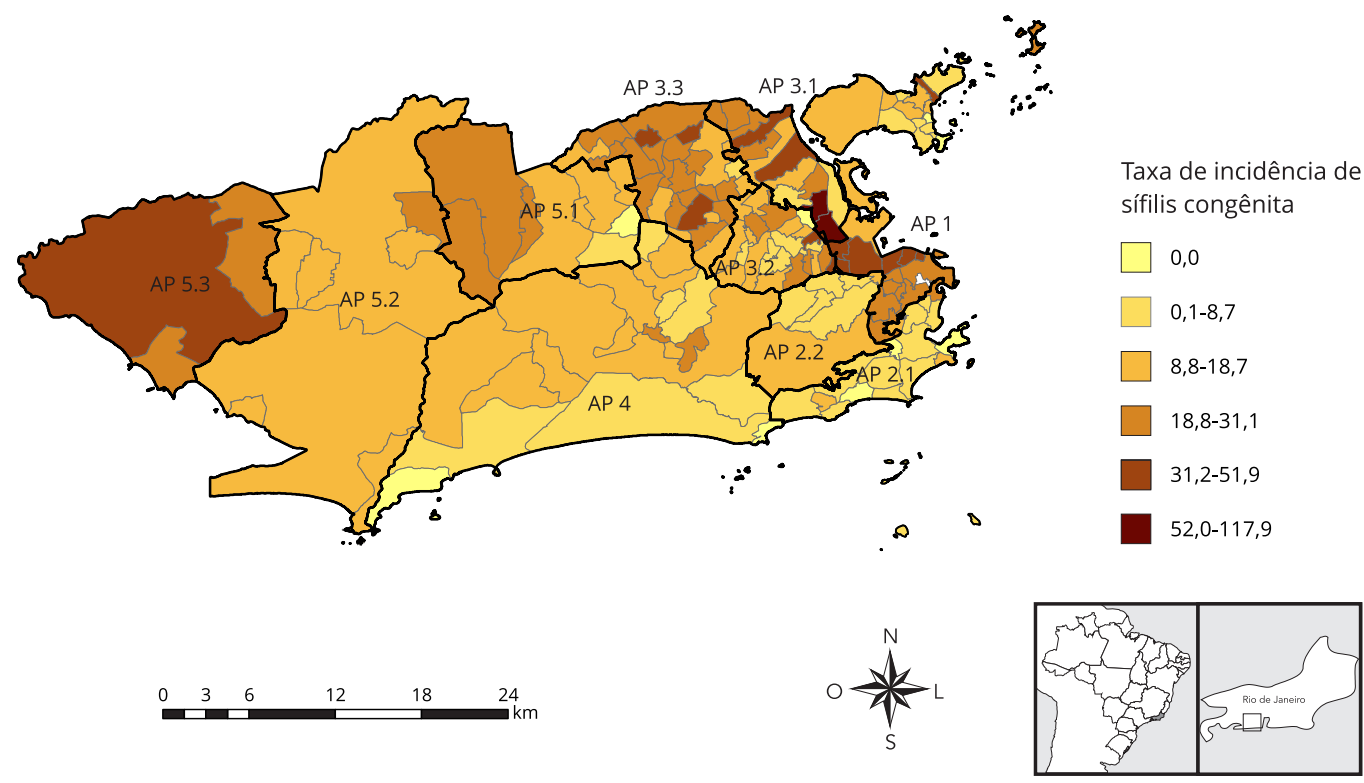

Fontes: Instituto Brasileiro de Geografia e Estatística; Sistema de Informação de Agravos de Notificação e o Sistema de Informaç̃̃es sobre Nascidos Vivos. 
Tabela 3

Variáveis explicativas e seus respectivos parâmetros.

\begin{tabular}{|c|c|c|c|c|c|c|}
\hline Variáveis explicativas/Parâmetros & $\begin{array}{l}\text { Coeficiente } \\
\text { Spearman }\end{array}$ & $\begin{array}{l}\text { Valor } \\
\text { mínimo }\end{array}$ & Média & $\begin{array}{l}\text { Valor } \\
\text { máximo }\end{array}$ & DP & $\mathrm{IC95 \%}$ \\
\hline Número de banheiros por morador & 0,63 & 0,28 & 0,52 & 1,27 & 0,19 & $0,49-0,55$ \\
\hline $\begin{array}{l}\text { Proporção de analfabetismo na população de } \\
10-14 \text { anos }\end{array}$ & 0,47 & 0,00 & 1,71 & 4,90 & 0,98 & $1,56-1,86$ \\
\hline $\begin{array}{l}\text { Rendimento médio dos responsáveis pelos } \\
\text { domicílios em salários mínimos }\end{array}$ & 0,63 & 1,18 & 4,65 & 24,02 & 3,86 & $4,05-5,25$ \\
\hline Proporção de gravidez na adolescência & 0,66 & 0,00 & 0,15 & 0,29 & 0,06 & $0,14-0,16$ \\
\hline $\begin{array}{l}\text { Proporção de nascidos vivos cuja mãe fez } 7 \\
\text { consultas ou mais no pré-natal }\end{array}$ & 0,71 & 0,45 & 0,72 & 0,97 & 0,10 & $0,70-0,74$ \\
\hline Densidade populacional de pobres & 0,48 & 0,00 & $6.337,88$ & $58.161,00$ & $6.084,12$ & $\begin{array}{l}5.386,19- \\
7.289,57\end{array}$ \\
\hline Proporção de população cuja cor da pele é parda & 0,67 & 0,02 & 0,11 & 0,28 & 0,05 & $0,10-0,12$ \\
\hline Proporção de população cuja cor da pele é preta & 0,53 & 0,07 & 0,35 & 0,65 & 0,12 & $0,33-0,37$ \\
\hline
\end{tabular}

DP: desvio padrão; IC95\%: intervalo de 95\% de confiança.

Fontes: Instituto Brasileiro de Geografia e Estatística e Secretaria Municipal de Saúde do Rio de Janeiro. Elaboração própria.

A técnica de análise da árvore de decisão ou regressão estabelece uma relação entre a variável desfecho e as oito variáveis explicativas selecionadas, resultando na previsão de um novo valor para a variável desfecho, baseada na relação estabelecida pelo algoritmo, com as variáveis explicativas. Observa-se que com a aplicação do modelo da árvore de regressão houve uma redução da variabilidade dos valores da taxa de incidência e que há correlação expressiva $(r=0,79)$ entre os valores (observados e previstos). A previsão de valores da variável desfecho, baseada na relação com as variáveis explicativas, permite a explicitação quantitativa do erro médio da taxa de incidência de sífilis congênita, decorrente da aplicação do modelo. O erro médio quadrático observado nas taxas é da ordem de 10,85 casos/mil nascidos vivos, e o erro médio no modelo foi da ordem de $11 \%$. Na Figura 2, observa-se a expressão gráfica da árvore de regressão.

Com o formato de árvore invertida, a referida técnica estabelece nós de partição entre as unidades de análise, os quais dão origem a grupos com o valor homogêneo da variável desfecho, sendo que o grupo após cada bifurcação à esquerda refere-se ao grupo de bairros que atingiram o corte estabelecido pelo algoritmo, e o grupo da direita não atingiu este valor de corte. As caixas apresentam três valores numéricos: na parte superior, o valor expressa a taxa média de sífilis congênita após a aplicação do modelo, o valor de $\mathrm{n}$ se refere ao número de bairros classificados nos respectivos grupos e o valor percentual diz respeito à proporção em relação às 157 unidades de análise (bairros).

O primeiro nó da árvore é estabelecido por meio de um corte na proporção de nascidos vivos, cuja mãe fez sete ou mais consultas de pré-natal. Nos 58 bairros onde essa proporção é maior que $73 \%$, a taxa de incidência média é de cerca de 7,1 casos por mil nascidos vivos. Nesse grupo de bairros, há outra partição utilizando a mesma variável, mas com ponto de corte de $82 \%$. Em 27 bairros a proporção foi superior ao corte estabelecido pelo algoritmo da árvore de regressão (82\%), e a taxa de incidência de sífilis congênita prevista neste grupo foi a menor $(3.2 ; \pm 2,7)$ por mil nascidos vivos, evidenciando a relevância da cobertura da assistência pré-natal na resposta ao problema.

Em 99 bairros não foi atingido o percentual de 73\% de nascidos vivos cuja mãe fez sete consultas ou mais de pré-natal, e a taxa de incidência média nos mesmos foi de aproximadamente 23/mil nascidos vivos. A partição desse grupo de bairros ocorreu por meio da mesma variável, no entanto, com um valor de corte menor, de $57 \%$. Os dez bairros que não atingiram essa proporção foram aqueles cuja previsão da taxa de incidência de sífilis congênita foi a maior - $38( \pm 8,7)$ casos/mil nascidos vivos. Os 10 bairros classificados nessa pior categoria são: Saúde, Gamboa, Santo Cristo, Cordovil, Acari, Barros Filho, Costa Barros, Cidade de Deus, Senador Camará e Jacarezinho. 
Figure 2

Árvore de regressão pelo método CaRT (Classification and Regression Trees) da taxa de incidência de sífilis congênita nos bairros do Município do Rio de Janeiro, Brasil.

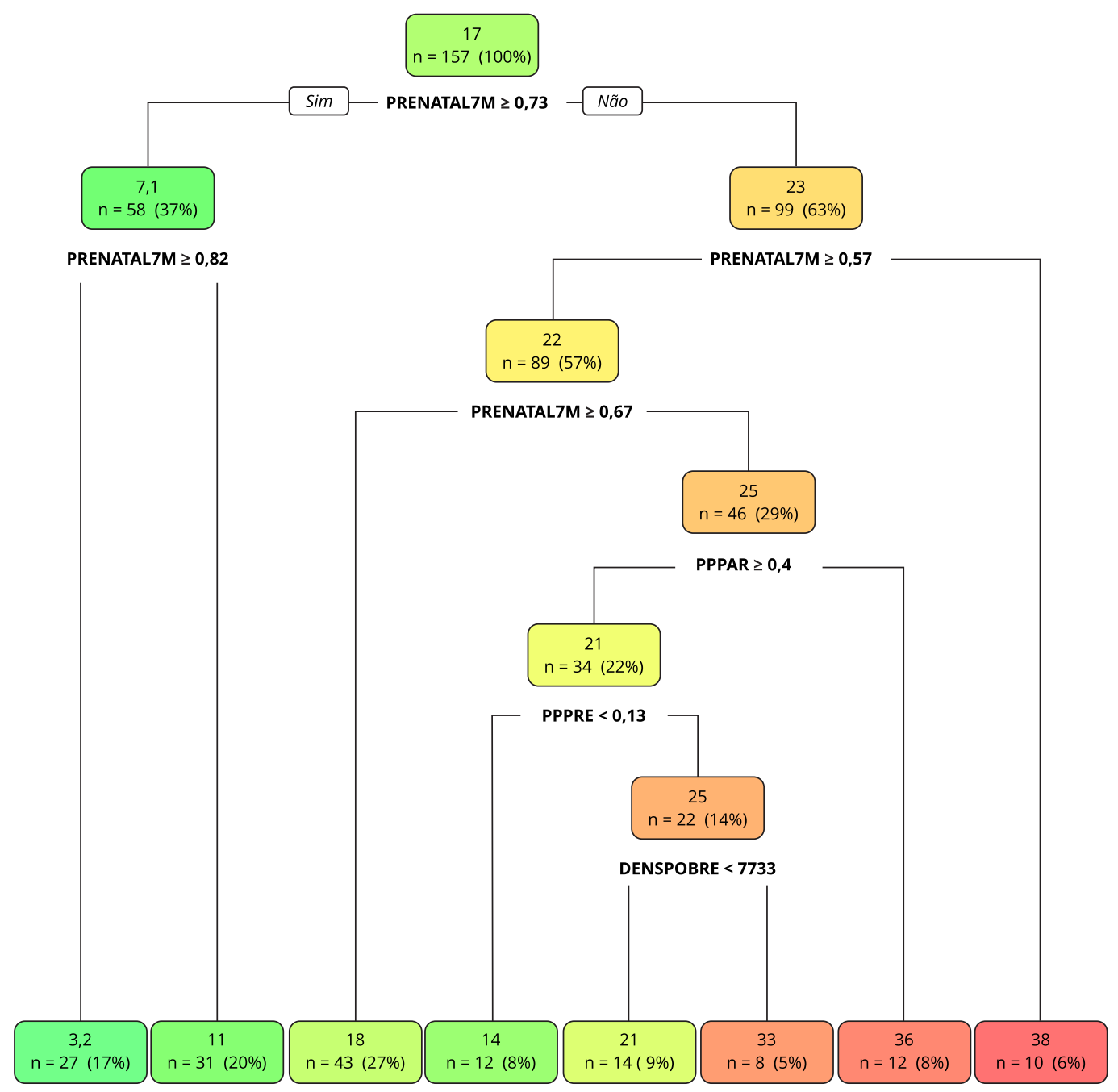

DENSPOBRE: densidade populacional de pobres; PPPAR: proporção da população cuja cor da pele é parda; PPPRE: proporção da população cuja cor da pele é preta; PRENATAL7M: proporção de nascidos vivos cuja mãe fez 7 consultas ou mais no pré-natal.

Os 89 bairros que atingiram a proporção de $57 \%$ de mães com sete consultas ou mais sofreram partição pela mesma variável, agora com corte de 67\%. Em 43 bairros essa proporção foi atingida e a taxa de incidência de sífilis congênita prevista foi de $18 /$ mil nascidos vivos. Os demais bairros (46) não atingiram essa proporção e sofreram partição pela variável proporção de população de pele parda, tendo como ponto de corte $40 \%$. Nos 12 bairros onde a proporção da população parda não foi superior a 40\%, a taxa de incidência prevista de sífilis congênita foi de aproximadamente $36 / \mathrm{mil}$ nascidos vivos.

Nos 34 bairros onde a proporção da população parda foi superior a $40 \%$ houve outra partição por meio da variável proporção da população de pele preta, tendo como ponto de corte 13\%. A taxa de incidência de sífilis congênita prevista no grupo com menos de 13\% de população negra foi inferior à média da cidade do Rio de Janeiro (cerca de 14/mil nascidos vivos). O grupo dos 25 bairros em que 
a proporção de população negra é superior a 13\% sofreu mais uma partição, utilizando-se a variável densidade de pobres, com ponto de corte de $7.733 / \mathrm{km}^{2}$. Nos 14 bairros onde a densidade de pobres foi inferior a esse ponto de corte, a taxa de incidência foi de 21 casos/mil nascidos vivos. Nos oito bairros em que a densidade de pobres foi superior a esse ponto, a taxa foi de 33/mil nascidos vivos.

\section{Discussão}

A condição de doença evitável da sífilis congênita pressupõe o diagnóstico e tratamento das gestantes infectadas, e neste sentido, os resultados apresentados evidenciam a influência das deficiências relacionadas ao sistema de saúde na persistência de patamares elevados da referida doença.

Em relação à descrição dos casos notificados de sífilis congênita, constatou-se que em $73 \%$ deles a mãe frequentou, ao menos, uma consulta (Tabela 2). Uma limitação importante desse dado reside no fato de que se trata de variável que não permite a estratificação dos casos de acordo com o número de consultas de pré-natal realizadas, isto é, refere-se a uma variável dicotômica. Embora a proporção que não frequentou o pré-natal seja minoritária (18\%), é uma proporção expressiva que representa 1.112 casos, cujas mães não obtiveram acesso à assistência pré-natal em nenhum momento de sua gestação.

Esse resultado está de acordo com a alta proporção de gestantes que tiveram diagnóstico tardio de sífilis. Segundo dados do SINAN, foi possível observar que em $50 \%$ dos casos notificados não houve diagnóstico oportuno para evitar o caso de sífilis congênita, visto que em $44 \%$ deles o diagnóstico da sífilis materna foi realizado no momento do parto (no caso de nascido vivo) ou na curetagem (no caso de abortos), em $5 \%$ dos casos o diagnóstico foi realizado após o parto e em $1 \%$ o diagnóstico de sífilis materna nem foi realizado. Esses dados evidenciam falha da atuação da vigilância epidemiológica, especificamente no que diz respeito à detecção oportuna dos casos de sífilis em gestantes.

Se a variável descrita anteriormente (momento de diagnóstico da sífilis materna) sugere falhas na capacidade de diagnosticar os casos de sífilis oportunamente, a informação sobre o tratamento da gestante $-86 \%$ dos casos com tratamento inadequado ou não realizado - evidencia os problemas relacionados a este cuidado. Em apenas $11 \%$ dos casos notificados de sífilis congênita o(s) parceiro(s) sexual(is) foi(ram) tratado(s). É importante que a atenção primária esteja estruturada para acolher e tratar esses parceiros das gestantes notificadas com sífilis, como forma de dar resposta adequada ao problema, evitando a reinfecção.

Em uma revisão conceitual sobre o acesso e a utilização de serviços de saúde foi constatado que a utilização, embora seja uma expressão do acesso, não se explica apenas por este aspecto. Além do acesso - que se relaciona com a oferta de serviços - atributos individuais e contextuais podem influenciar a utilização dos serviços de saúde 20 . Nesse sentido, pode-se afirmar que a utilização dos serviços de pré-natal é influenciada por características socioeconômicas dos indivíduos e de seus contextos de vida, e pelo acesso, que está vinculado à oferta de serviços de atenção primária.

No que diz respeito à escolaridade, trata-se de uma variável relevante para a caracterização socioeconômica dos indivíduos 21 , no entanto, a distribuição dos casos por esta variável evidencia que em $41 \%$ dos casos não há informação da escolaridade da mãe. Observou-se que dentre os casos que tiveram o registro dessa variável na ficha de notificação, a maioria deles tem escolaridade até o Nível Fundamental. Lima et al. 8, Melo et al. 10 e Araújo et al. 11 identificaram maior incidência de sífilis congênita na população com baixa escolaridade, em distintas unidades de análise - Recife, Pernambuco (2004-2006), Belo Horizonte, Minas Gerais, (2001-2008) e Brasil (2008) respectivamente.

A proporção de casos cuja cor da pele não é preenchida é extremamente elevada (42\%), prejudicando a interpretação da distribuição dos casos por tal variável. Ainda que a baixa qualidade dos dados apresentados seja um empecilho para a interpretação dos mesmos, e considerando que a proporção da população branca na cidade do Rio de Janeiro é de 51\% (Secretaria Municipal de Saúde, http:// tabnet.rio.rj.gov.br/), destaca-se a baixa proporção de casos cuja cor da pele é branca (15\%). Tal resultado corrobora os resultados de Lima et al. 8, Melo et al. 10 e Araújo et al. 11, que identificaram maior incidência de sífilis congênita na população preta e parda.

No que tange à mortalidade, destaca-se que no período estudado ocorreram 120 óbitos (aproximadamente $2 \%$ do total), 364 abortos (6\%) e 315 natimortos (5\%). São valores expressivos de mortes 
evitáveis. A permanência dessa situação é considerada inaceitável, indicando a necessidade de priorização da resposta do setor saúde ao problema. Em relação à mortalidade por sífilis congênita, ressalta-se que, entre 1999 e 2002, a taxa de mortalidade perinatal geral reduziu no Município do Rio de Janeiro, entretanto, a mortalidade específica por sífilis congênita permaneceu estável 5 .

A análise da sífilis congênita nos bairros da cidade evidenciou a relevância da variável proporção de nascidos vivos cuja mãe fez sete consultas ou mais no pré-natal como componente importante para a explicação do problema. Trata-se de variável que expressa a importância da continuidade do cuidado, característica central da atenção primária em saúde 22 na detecção e tratamento das gestantes infectadas, assim como seu(s) parceiro (s) sexual(is), além do monitoramento de possíveis reinfecções, evitando a transmissão vertical da sífilis.

Uma resposta efetiva à sífilis congênita passa pela perspectiva da integralidade. Mattos 23 descreveu três sentidos da integralidade da atenção à saúde: como princípio das práticas cotidianas dos profissionais de saúde; como diretriz organizativa do sistema; e como resposta governamental aos problemas no campo da política de saúde 22.

A perspectiva da integralidade como princípio das práticas cotidianas dos profissionais de saúde envolve uma atitude crítica à fragmentação do cuidado. Nesse sentido, um aspecto a ser enfrentado é a baixa frequência ao tratamento do(s) parceiro(s) sexual(is). Trata-se da constatação que merece uma investigação sobre a maneira como os serviços e profissionais de atenção primária à saúde atuam no acolhimento e assistência voltados para os homens ao longo do período da gestação.

Do ponto de vista da organização das práticas, a integralidade indica a necessidade de definição, por parte do poder público municipal - que tem competência na organização da atenção primária em saúde, no contexto do SUS - de diretrizes terapêuticas, compromisso dos profissionais da atenção primária no cuidado, tais como a definição de responsabilidades pela solicitação de testes diagnósticos, fluxos para tratamento, unidades de saúde de referência em casos de resposta alérgica ao tratamento com a penicilina.

No que tange à organização dos serviços de vigilância em saúde, pode-se atuar para além dos processos de diagnóstico (detecção) e registro (notificação), avançando na produção de estudos sobre análises de situação de saúde locais, buscando identificar a dinâmica cotidiana existente no território, no sentido de subsidiar o planejamento em saúde e fomentar a participação popular. Ainda que as ações de vigilância epidemiológica voltadas para a identificação dos casos para tomar medidas de controle sejam importantes, é fundamental avançar para a atuação de vigilância em saúde que esteja articulada aos processos sociais que ocorrem no território e integrada à atenção primária em saúde.

Na perspectiva da integralidade atrelada à política de saúde, urge a priorização da resposta à sífilis. Considerando-se a relevância da proporção de gestantes que realizam ao menos as sete consultas de pré-natal para explicação da taxa de incidência de sífilis congênita nos bairros da cidade do Rio de Janeiro, a priorização de ações para a expansão de oferta de serviços de saúde em algumas áreas é necessária. Os bairros localizados nas AP 1, 3.1 e 3.3 apresentam elevada incidência de sífilis congênita, expressando as necessidades de saúde destes territórios, que devem ser priorizados para a elevação da cobertura de consultas de pré-natal.

A recente expansão de cobertura das equipes de saúde da família no Município do Rio de Janeiro - de 3,5\% da população em 2008 para 51,7\% ao final de 201624 - é fundamental para uma resposta efetiva ao problema da sífilis congênita. No entanto, a cobertura ainda é insuficiente, sendo necessária a continuidade desse processo de expansão, sobretudo nas áreas com cobertura baixa e de altas incidências.

Ainda que a influência das falhas dos serviços de saúde esteja bastante evidente na transmissão vertical da sífilis, não se pode ignorar a relevância das condições de vida neste processo. Os resultados apresentados na análise das taxas de incidência de sífilis congênita nos bairros da cidade do Rio de Janeiro evidenciam a relevância das variáveis sobre condições de vida - proporção da população cuja cor da pele é parda ou preta e densidade de pobres - na explicação do risco de transmissão vertical da sífilis. É importante que o planejamento em saúde leve em consideração esses aspectos, para que a resposta ao problema seja aprimorada.

Este trabalho abre perspectivas para novos estudos utilizando abordagens preditivas sobre as bases de dados relacionadas a sífilis congênita e seus determinantes sociais, por meio da adoção de técnicas de ciência de dados aplicadas à saúde. As limitações do trabalho estão relacionadas à qualidade 
de alguns dados. Ao utilizar dados secundários, assume-se os limites decorrentes das condições técnico-operacionais da vigilância epidemiológica municipal na produção dos dados sobre os casos de sífilis congênita 15 . Outra limitação que merece ser citada é que o estudo não abordou o problema de abastecimento de penicilina 25 , único medicamento indicado para o tratamento da sífilis em gestante e, portanto, uma medida para evitar a transmissão vertical. A ausência de informações sobre a disponibilidade (e dispensação) da penicilina nos serviços de saúde da cidade do Rio de Janeiro impossibilitou a análise do impacto da falta do medicamento na incidência da sífilis congênita.

Este artigo ilustra que os modelos CaRT podem lidar com vários tipos de resultados, mas de maneira diferente dos modelos de regressão. As intervenções, desenvolvidas baseando-se nos resultados do modelo de regressão, são voltadas para o membro médio da população, sem considerar os subgrupos de população como o principal alvo. Uma análise CaRT tem a capacidade de segmentar eficientemente as populações em subconjuntos significativos, permitindo que os profissionais identifiquem segmentos de populações marginalizadas, direcionando de maneira efetiva a distribuição de recursos de saúde pública 26.

\section{Colaboradores}

G. J. Reis contribuiu na elaboração do projeto, da concepção do trabalho, interpretação e análise dos dados. C. Barcellos e M. M. Pedroso contribuíram substancialmente na concepção do trabalho, interpretação e análise dos dados. D. R. Xavier contribuiu substancialmente na análise dos dados. Todos os autores revisaram e aprovaram a versão final a ser publicada.

\section{Agradecimentos}

À Escola Nacional de Saúde Pública Sergio Arouca (ENSP), sobretudo aos professores do Departamento de Endemias, ao Laboratório de Geoprocessamento do Instituto de Comunicação Científica e Tecnológica em Saúde (ICICT) e à Fundação Oswaldo Cruz (Fiocruz).

\section{Referências}

1. Coordenação-Geral de Desenvolvimento da Epidemiologia em Serviços, Secretaria de Vigilância em Saúde, Ministério da Saúde. Guia de vigilância em saúde. Brasília: Ministério da Saúde; 2017.

2. Programa Nacional de DST/AIDS, Secretaria de Vigilância em Saúde, Ministério da Saúde. Diretrizes para controle da sífilis congênita: manual de bolso. 2a Ed. Brasília: Ministério da Saúde; 2006.

3. Wijesooriya NS, Rochat RW, Kamb ML, Turlapati P, Temmerman M, Broutet N, et al. Global burden of maternal and congenital syphilis in 2008 and 2012: a health systems modelling study. Lancet Glob Health 2016; 4:e525-33.

4. Ministério da Saúde. Indicadores e dados básicos da sífilis nos municípios brasileiros. http:// indicadoressifilis.aids.gov.br/ (acessado em 11/Jan/2017). 
5. Saraceni V, Guimarães MHFS, Theme Filha MM, Leal MC. Mortalidade perinatal por sífilis congênita: indicador da qualidade da atenção à mulher e à criança. Cad Saúde Pública 2005; 21:1244-50.

6. De Lorenzi DRS, Madi JM. Sífilis congênita como indicador de assistência pré-natal. Rev Bras Ginecol Obstet 2001; 23:647-52.

7. Domingues RMSM, Saraceni V, Hartz ZMDA, Leal MDC. Sífilis congênita: evento sentinela da qualidade da assistência pré-natal. Rev Saúde Pública 2013; 47:147-57.

8. Lima MG, Santos RFR, Barbosa GJA, Ribeiro GS. Incidência e fatores de risco para sífilis congênita em Belo Horizonte, Minas Gerais, 2001-2008. Ciênc Saúde Coletiva 2013; 18:499-506.

9. Lima BGC, Costa MCN, Dourado MIC. Avaliação da qualidade do rastreamento de HIV/ aids e sífilis na assistência pré-natal. Epidemiol Serv Saúde 2008; 17:125-7.

10. Melo NGDO, Melo Filho DA, Ferreira LOC. Diferenciais intraurbanos de sífilis congênita no Recife, Pernambuco, Brasil (2004-2006). Epidemiol Serv Saúde 2011; 20:213-22.

11. Araújo CL, Shimizu HE, Sousa AIA, Hamann EM. Incidência da sífilis congênita no Brasil e sua relação com a Estratégia Saúde da Família. Rev Saúde Pública 2012; 46:479-86.

12. Domingues RMSM, Saraceni V, Hartz ZMDA, Leal MDC. Sífilis congênita: evento sentinela da qualidade da assistência pré-natal. Rev Saúde Pública 2013; 47:147-57.

13. Barcellos C, Sabroza PC, Peiter P, Rojas LI. Organização espacial, saúde e qualidade de vida: análise espacial e uso de indicadores na avaliação de situações de saúde. Inf Epidemiol SUS 2002; 11:129-38.

14. Barcellos C, Santos SM. Colocando dados no mapa: a escolha da unidade espacial de agregação e integração de bases de dados em saúde e ambiente através do geoprocessamento. Inf Epidemiol SUS 1997; 6:21-9.

15. Rede Interagencial de Informação para a Saúde. Indicadores básicos para a saúde no Brasil: conceitos e aplicações. 2a Ed. Brasília: Organização Pan-Americana da Saúde; 2008.
16. Cavallieri F, Lopes GP. Índice de Desenvolvimento Social - IDS: comparando as realidades microurbanas da Cidade do Rio de Janeiro. Rio de Janeiro: Instituto Municipal de Urbanismo Pereira Passos; 2008.

17. Chiavegatto ADP. Uso de big data em saúde no Brasil: perspectivas para um futuro próximo. Epidemiol Serv Saúde 2015; 24:325-32.

18. Rodrigues MAS. Árvores de classificação [Monografia de Graduação]. Ponta Delgada: Universidade dos Açores; 2006.

19. Loh W-Y. Classification and regression trees. WIREs Data Mining Knowledge Discovery 2011; 1:14-23.

20. Travassos C, Martins M. Uma revisão sobre os conceitos de acesso e utilização de serviços de saúde. Cad Saúde Pública 2004; 20 Suppl 2:S190-8.

21. Galobardes B. Indicators of socioeconomic position (part 1). J Epidemiol Community Health 2006; 60:7-12.

22. Cunha EM, Giovanella L. Longitudinalidade/ continuidade do cuidado: identificando dimensões e variáveis para a avaliação da atenção primária no contexto do sistema público de saúde brasileiro. Ciênc Saúde Coletiva 2011; 16:1029-42.

23. Mattos RA. Os sentidos da integralidade: algumas reflexões acerca de valores que merecem ser defendidos. In: Pinheiro R, Mattos RA, organizadores. Os sentidos integralidade na atenção e no cuidado à saúde. Rio de Janeiro: Instituto de Medicina Social, Universidade do Estado do Rio de Janeiro/Abrasco; 2006.

24. Departamento de Atenção Básica, Ministério da Saúde. Histórico de cobertura da saúde da família. https://egestorab.saude.gov.br/pagi nas/acessoPublico/relatorios/relHistoricoCo bertura.xhtml (acessado em 21/Jan/2018).

25. Conselho Nacional de Secretários de Saúde. Contrato organizativo de ação pública da saúde - COAP. Brasília: Conselho Nacional de Secretários de Saúde; 2015. (Nota Técnica 03/2015).

26. Speybroeck N. Classification and regression trees. Int J Public Health 2012; 57:243-6. 


\section{Abstract}

The study aimed to characterize notified cases of congenital syphilis from 2011 to 2014 in the city of Rio de Janeiro and to analyze possible associations between congenital syphilis and living conditions in the city's neighborhoods. Cases of congenital syphilis were characterized according to biological and socioeconomic variables and health services use. At the aggregate level, regression tree technique was used for the data analysis, with mean incidence rate (2011-2014) of congenital syphilis as the dependent variable and housing quality, schooling, income, teenage pregnancy, poverty density, access to prenatal care, and skin color as independent variables. The dependent variable was mapped to identify spatial patterns. The SINAN, SINASC, and IBGE databases were used for notifiable diseases, live births, and census data, respectively. A total of 6,274 cases of congenital syphilis were reported, which represents an incidence rate of 17.3 cases $/ 1,000$ live births. Cases were distributed in the central, northern peripheral, and western zones of the city, with a high proportion of cases in infants of black mothers with low schooling. There was also a high proportion of pregnant women with late diagnosis of syphilis and inadequate treatment. At the aggregate level, the most relevant variable for explaining the problem was the low proportion of pregnant women with at least 7 prenatal visits. The analysis allowed the identification of marginalized population segments and can help direct public health resources more effectively.

Congenital Syphilis; Prenatal Care; Social Conditions; Sexually Transmitted Diseases

\section{Resumen}

El objetivo de este estudio fue caracterizar los casos notificados de sífilis congénita, durante el periodo de 2011 a 2014, en el municipio de Río de Janeiro, analizar posibles asociaciones entre la morbilidad por sífilis congénita, y las condiciones de vida de las poblaciones residentes en los barrios de la ciudad. Los casos de sifilis congénita fueron caracterizados de acuerdo a variables biológicas, socioeconómicas y de utilización de servicios de salud. En el nivel agregado, se utilizó el árbol de regresión como técnica de análisis de datos, considerando la tasa de incidencia media (2011-2014) de sífilis congénita como variable dependiente, e indicadores relativos a la calidad habitacional, educación, renta, embarazo en la adolescencia, densidad de pobres, acceso a la asistencia prenatal y color de piel como variables independientes. Hubo un mapeo de la variable dependiente para la identificación de patrones espaciales. Se utilizaron datos del SINAN, SINASC e IBGE. Se notificaron 6.274 casos de sifilis congénita, lo que representa una tasa de incidencia de 17,3 casos/1.000 nacidos vivos. Los casos se distribuyen preponderantemente en la zona central, norte suburbana y oeste de la ciudad, con una elevada proporción de casos con baja escolaridad y con color de piel negro. Se observó una alta proporción de gestantes que tuvieron un diagnóstico tardío de sífilis y tratamiento inadecuado. En el nivel agregado, la variable más relevante para la explicación de los problemas fue la baja proporción de gestantes que frecuentaron, al menos, 7 consultas de asistencia prenatal. El análisis permitió la identificación de segmentos de poblaciones marginadas, pudiendo dirigir de manera efectiva la distribución de recursos de salud pública.

Sífilis Congénita; Atención Prenatal; Condiciones Sociales; Enfermedades de Transmisión Sexualmente
Recebido em 21/Jun/2017

Versão final reapresentada em 11/Abr/2018

Aprovado em 19/Abr/2018 